Pontifícia Universidade $_{\text {Do Rio de Janeiro }}$

Omar Paranaiba Vilela Neto

Simulação e Síntese Automática de Circuitos de Autômatos Celulares com Pontos Quânticos Através de Técnicas Inteligentes

Dissertação de Mestrado

Dissertação apresentada como requisito parcial para obtenção do título de Mestre pelo Programa de PósGraduação em Engenharia Elétrica da PUC-Rio.

Orientadores: Marco Aurélio C. Pacheco

Carlos Roberto H. Barbosa 
Pontifícia Universidade $_{\text {Do Rio de Janeiro }}$

Omar Paranaiba Vilela Neto

\title{
Simulação e Síntese Automática de Circuitos de Autômatos Celulares com Pontos Quânticos Através de Técnicas Inteligentes
}

Dissertação apresentada como requisito parcial para obtenção do título de Mestre pelo Programa de PósGraduação em Engenharia Elétrica da PUC-Rio. Aprovada pela Comissão Examinadora abaixo assinada.

\author{
Marco Aurélio C. Pacheco \\ Orientador \\ PUC-Rio \\ Carlos Roberto Hall Barbosa \\ Orientador \\ PUC-Rio \\ Rodrigo Prioli \\ PUC-Rio \\ Renato Portugal \\ LNCC \\ Maurício Pamplona \\ UFRJ \\ Prof. José Eugenio Leal
} Coordenador(a) Setorial do Centro Técnico Científico - PUC-Rio 
Todos os direitos reservados. É proibida a reprodução total ou parcial do trabalho sem autorização da universidade, do autor e do orientador.

\section{Omar Paranaiba Vilela Neto}

Graduou-se em Engenharia de Computação na Pontifícia Universidade Católica do Rio de Janeiro em 2004.

Ficha Catalográfica

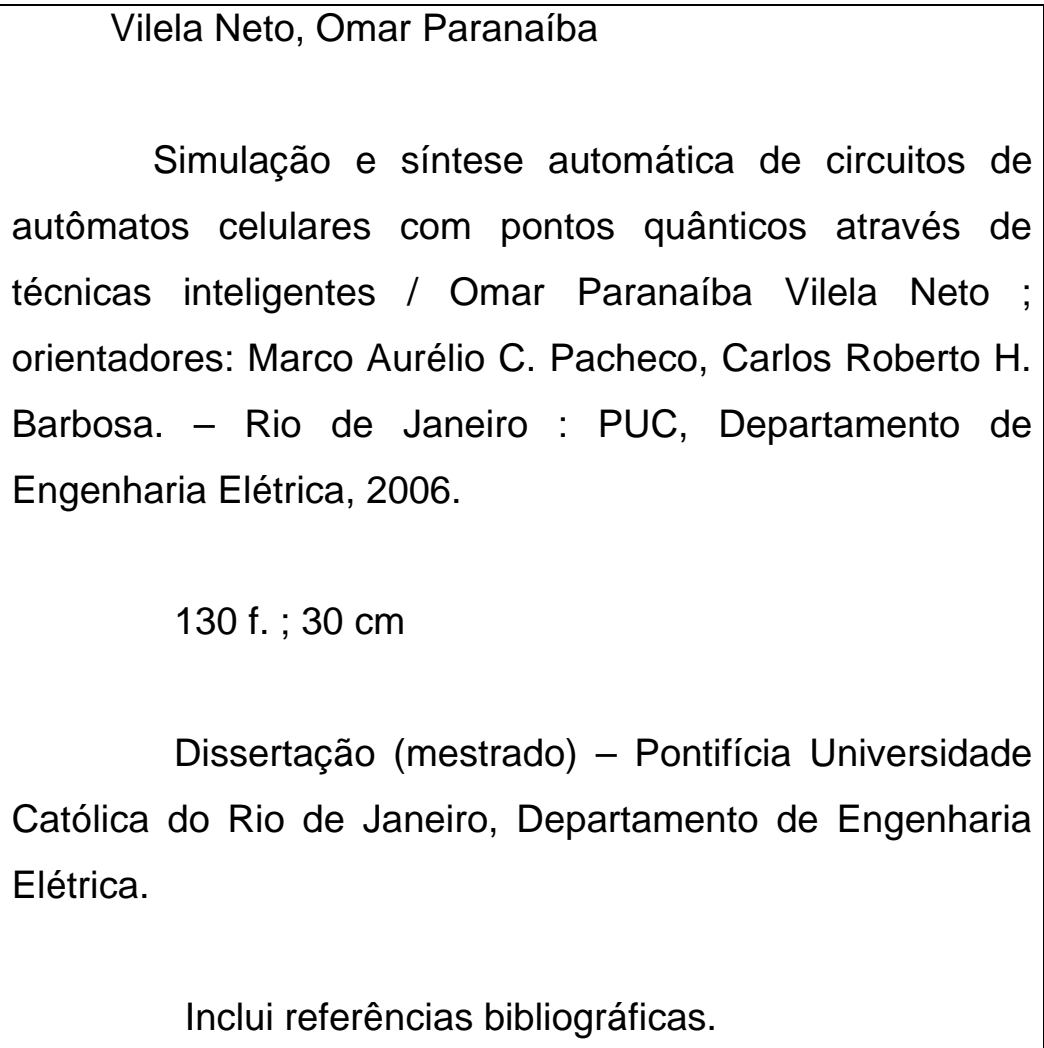

Simulação e síntese automática de circuitos de autômatos celulares com pontos quânticos através de técnicas inteligentes / Omar Paranaíba Vilela Neto ; orientadores: Marco Aurélio C. Pacheco, Carlos Roberto H. Barbosa. - Rio de Janeiro : PUC, Departamento de Engenharia Elétrica, 2006.

130 f. ; $30 \mathrm{~cm}$

Dissertação (mestrado) - Pontifícia Universidade Católica do Rio de Janeiro, Departamento de Engenharia Elétrica.

Inclui referências bibliográficas.

1. Engenharia elétrica - Teses. 2. Nanotecnologia. 3. Nanoeletrônica. 4. Pontos Quânticos. 5. Autômatos Celulares. 6. Rede Neurais. 7. Algoritmos Genéticos. I. Pacheco, Marco Aurélio C. II. Barbosa, Carlos Roberto H. III. Pontifícia Universidade Católica do Rio de Janeiro. Departamento de Engenharia Elétrica. IV. Título. 


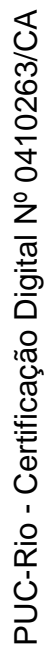

Aos meus pais Cora Alice e Paulo Antônio. 


\section{Agradecimentos}

Ao CNPq pelo apoio financeiro

Aos meus orientadores Professores Marco Aurélio C. Pacheco e Carlos Roberto Hall Barbosa pelo apoio e parceria neste trabalho.

Aos demais professores pelos ensinamentos.

Aos amigos Juan Lazo Lazo, Yván Jesus Túpac Valdivia e André Vargas Abs da Cruz pelo apoio e comentários durante o desenvolvimento.

Aos amigos Leone Masiero e Richardson Sadoco pelo suporte e apoio.

Aos amigos e familiares que torceram por mim nestes dois anos.

Aos funcionários da oficina de manutenção do departamento de elétrica pela ajuda.

Aos amigos do ICA por seu contínuo apoio e colaboração.

À minha irmã, cunhado e sobrinho pelo apoio e confiança.

À Adrissa pelo amor, auxílio e paciência. 


\section{Resumo}

Omar Paranaiba Vilela Neto. Simulação e Síntese Automática de Circuitos de Autômatos Celulares com Pontos Quânticos Através de Técnicas Inteligentes. Rio de Janeiro, 2006. 130p. Dissertação de Mestrado - Departamento de Engenharia Elétrica, Pontifícia Universidade Católica do Rio de Janeiro.

Esta dissertação investiga e propõe um novo simulador de circuitos de Autômatos Celulares com Pontos Quânticos (QCA) e uma nova metodologia para a criação e otimização de circuitos lógicos, utilizando técnicas da inteligência computacional. Autômatos Celulares com Pontos Quânticos é uma nova tecnologia, na escala nanométrica, que tem chamado a atenção dos pesquisadores por ser uma alternativa à tecnologia CMOS, cujo limite físico de miniaturização será atingido nos próximos anos. QCA tem um grande potencial no desenvolvimento de circuitos com maior densidade espacial, maior velocidade, baixa dissipação e baixo consumo de energia. Ao contrário das tecnologias tradicionais, QCA não codifica a informação pelo fluxo de corrente elétrica, mas pela configuração das cargas elétricas no interior das células. A interação coulombiana entre as células garante o fluxo da informação. Apesar de simples, essas características fazem com que a arquitetura de circuitos de QCA se torne não trivial. Portanto, a criação de um simulador e de uma metodologia de elaboração e síntese automática de circuitos possibilitam aos cientistas uma melhor visualização de como esses dispositivos funcionam, acelerando o desenvolvimento desses sistemas na escala nanométrica. Para atingir o objetivo proposto, técnicas de inteligência computacional, tais como redes neurais do tipo Hopfield, para o desenvolvimento do simulador, e algoritmos genéticos, para a metodologia de criação e otimização dos circuitos, foram empregadas. Os resultados encontrados foram significativos, comprovando que as técnicas da inteligência computacional podem ser uma ferramenta estratégica para o rápido desenvolvimento da nanoeletrônica e da nanotecnologia em geral.

\section{Palavras-chave}

Nanotecnologia, Nanoeletrônica, Pontos Quânticos, Autômatos Celulares, Rede Neurais, Algoritmos Genéticos. 


\section{Abstract}

Omar Paranaiba Vilela Neto. Simulation and Automatic Syntheses of Quantum Dots Cellular Automata circuits thought Intelligent Techniques. Rio de Janeiro, 2006. 130p. MSc. Dissertation - Department of Electrical Engineering, Pontifical Catholic University of Rio de Janeiro.

This dissertation investigates and considers a new simulator of Quantum Dots Cellular Automata (QCA) Circuits and a new methodology for the synthesis and optimization of logical circuits, by means of Computational Intelligence. Quantum-dot Cellular Automata (QCA) is a new technology in the nanometric scale which has called attention from researchers as one alternative for the CMOS technology, which is reaching its physical limitation. QCA have a large potential in the development of circuits with high space density and low heat dissipation, and can allow the development of faster computers with lower power consumption. Differently from the conventional technologies, QCA do not codify information by means of electric current flow, but rather by the configuration of electrical charges in the interior of the cells. The Coulomb interaction between cells is responsible by the flow of information. Despite simple, these features become the design of logical devices into a non-trivial task. Therefore, the development of a simulator and a methodology of automatic synthesis of QCA circuits make possible to the scientist a better evaluation of how these circuits work, accelerating the development of these new systems in the nanometer scale. To reach the proposed target, Computational Intelligence techniques were used. The first results show that these techniques are capable of simulating efficiently and fast, synthesizing optimized circuits with a reduced number of cells. Such optimization reduces the possibility of failures and guarantees higher speed.

\section{Keywords}

Nanotechnology, Nanoelectronic, Quantum Dots, Cellular Automata, Neural Network, Genetic Algorithm. 


\section{Sumário}

1 Introdução 17

$\begin{array}{ll}\text { 1.1. Motivação } & 17\end{array}$

1.2. Objetivos 19

1.3. Descrição do Trabalho 19

1.4. Organização da Dissertação 20

2 Inteligência Computacional no Apoio à Nanotecnologia 22

2.1. Inteligência Computacional 23

2.1.1. Algoritmos Genéticos 23

2.1.2. Lógica Fuzzy 24

2.1.3. Redes Neurais 26

2.1.4. Sistemas Híbridos 26

2.2. Sistema de Apoio à Nanotecnologia $\quad 27$

3 Autômatos Celulares com Pontos Quânticos (QCA) 33

3.1. Introdução 33

3.2. Princípios dos Dispositivos 33

3.3. Dispositivos Lógicos Básicos 37

3.4. Células Rotacionadas e Circuitos em um Plano 39

3.5. Zonas de Clock 40

3.6. Circuitos Lógicos já Desenvolvidos 43

3.7. Alternativas de Fabricação 43

3.7.1. Ilhas de Metal 44

3.7.2. Semicondutor 44

3.7.3. Magnético 44

3.7.4. Molecular 45

4 Simulador de QCA $\quad 46$

4.1. Introdução 46

4.2. Simuladores de QCA Já Existentes 46 
4.3. Rede Neural do Tipo Hopfield 48

4.4. Considerações Sobre os Dispositivos Simulados 49

4.4.1. Raio de Vizinhança de uma Célula 50

4.4.2. Interação entre as Células $\quad 51$

4.5. A Simulação 56

4.6. Resultados $\quad 59$

4.6.1. Majority Gate $\quad 59$

$\begin{array}{ll}\text { 4.6.2. Circuito com ruído } & 61\end{array}$

5 Síntese Automática de Circuitos de QCA por Algoritmos Genéticos 64

5.1. Descrição do Problema 64

5.2. Representação dos Indivíduos 66

5.3. Avaliação dos Indivíduos 70

6 Estudos de Casos $\quad 72$

6.1. Porta Lógica OU de 4 Entradas 72

6.2. Multiplexador 76

6.3. Ou-exclusivo 79

6.4. Somador Completo 82

6.4.1. Alternativa 1

6.4.2. Alternativa $2 \quad 85$

7 Conclusões e Trabalhos Futuros $\quad 88$

8 Referência Bibliográficas 91

$\begin{array}{ll}\text { Apêndice } 1 & 97\end{array}$

$\begin{array}{ll}\text { Células Rotacionadas Lado a Lado } & 97\end{array}$

Uma Célula Convencional e uma Célula Rotacionada Lado a Lado $\quad 97$

Células Convencionais Distanciadas de Meia Célula 98

Células Rotacionadas Distanciadas de Meia Célula 98

Uma Célula Convencional e uma Célula Rotacionada Distanciadas de $\begin{array}{ll}\text { Meia Célula } & 99\end{array}$ 
Células Convencionais Distanciadas de Uma Célula

Células Rotacionadas Distanciadas de Uma Célula

Uma Célula Convencional e uma Célula Rotacionada Distanciadas de

Uma Célula

Células Convencionais em Diagonal 101

Células Rotacionadas em Diagonal 101

Uma Célula Convencional e uma Célula Rotacionada em Diagonal 102

Células Convencionais em Diagonal Distanciadas 102

$\begin{array}{ll}\text { Células Rotacionadas em Diagonal Distaciadas } & 103\end{array}$

Uma Célula Convencional e uma Célula Rotacionada em Diagonal

Distanciadas

Células Convencionais em Meia Diagonal 104

Células Rotacionadas em Meia Diagonal 105

Uma Célula Convencional e uma Célula Rotacionada em Meia

Diagonal

Células Convencionais em Meia Diagonal Distanciadas 106

Células Rotacionadas em Meia Diagonal Distanciada 107

Uma Célula Convencional e uma Célula Rotacionada em Meia

Diagonal Distanciadas

Apêndice 2

Inversor

109

Dois Fios Cruzando em um Plano 110

Majority Gate Tradicional 111

$\begin{array}{ll}\text { Porta Lógica } E & 113\end{array}$

Majority Gate Alterado 113

$\begin{array}{ll}\text { Somador Completo } & 117\end{array}$

$\begin{array}{ll}\text { Apêndice } 3 & 123\end{array}$

$\begin{array}{ll}\text { Porta OU de } 4 \text { entradas } & 123\end{array}$

$\begin{array}{ll}\text { Multiplexador } & 124\end{array}$

OU Exclusivo 126

Somador Completo - Alternativa 1

$\begin{array}{ll}\text { Somador Completo - Alternativa } 2 & 128\end{array}$ 


\section{Lista de figuras}

Figura 1- Procedimento básico do algoritmo genético..................................... 24

Figura 2- Componentes de um Conjunto Fuzzy............................................... 25

Figura 3- Diagrama de uma célula de QCA com quatro pontos quânticos em seus dois possíveis estados de polarização........................................................... 34

Figura 4- Interação não-linear entre as células de QCA. A polarização da célula 2 é fixa e influencia a polarização da célula 1 ................................................. 34

Figura 5- Duas células lado a lado com polarizações semelhantes e com

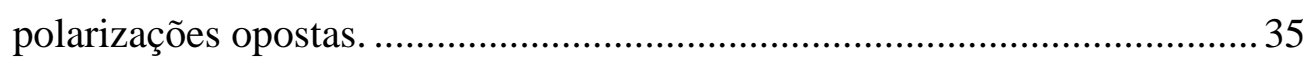

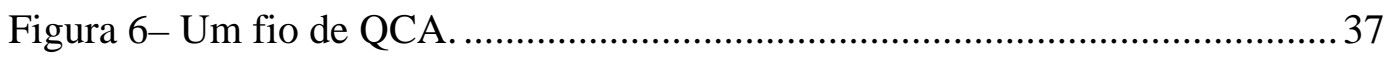

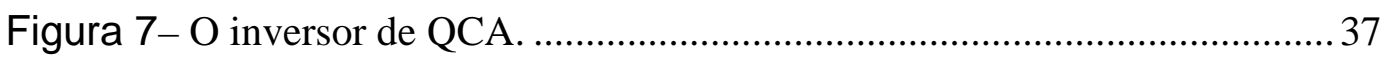

Figura 8- O a distribuição de sinal em um circuito de QCA. .............................. 38

Figura 9- O Majority Gate e como ele pode ser usado para implementar as portas

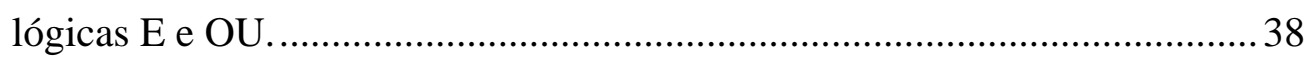

Figura 10- Um fio de células rotacionadas....................................................... 39

Figura 11- Interação coulombiana entre uma célula tradicional e uma

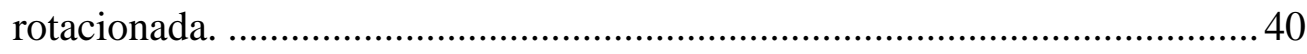

Figura 12- Cruzamento de informações em um plano. ....................................... 40

Figura 13- As quatro fases de clock em QCA................................................ 41

Figura 14- Majority Gate incorreto.............................................................. 42

Figura 15-Majority Gate com as zonas de clock sincronizando a informação... 42

Figura 16- Estrutura de uma rede neural do tipo Hopfield com conexões entre

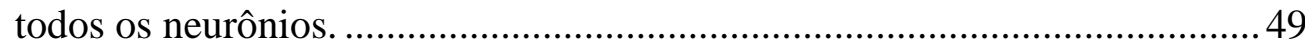

Figura 17- Exemplo de grade para posicionamento de células........................... 50

Figura 18- O raio de vizinhança de uma célula de QCA do simulador............... 50

Figura 19- Duas células lado a lado com a polarizações diferentes e polarizações

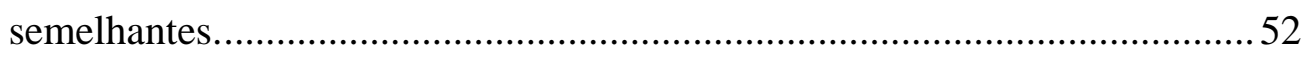

Figura 20- Como a posição da célula inverte o sinal da "kink energy” quando uma célula convencional é vizinha de uma célula rotacionada.....................56

Figura 21- O circuito do Majority Gate (os tons de cinza representam as 
diferentes zonas de clock). E a topologia da rede neural de Hopfield que o representa.

Figura 22- Algoritmo simplificado utilizado na simulação 58

Figura 23- O circuito do Majority Gate (os tons de cinza representam as diferentes zonas de clock). E a topologia da rede neural de Hopfield que o representa. 59

Figura 24- Resultado da simulação do majority gate para um conjunto de entradas. 61

Figura 25- Arranjo de células com ruído. À direita o resultado da simulação, onde a cor vermelho representa a polarização -1 e a cor azul a polarização 1 .

Figura 26-- Arranjo de células sem ruído. À direita o resultado da simulação, onde a cor vermelho representa a polarização -1 e a cor azul a polarização 1 .

Figura 27- Modelo co-evolucionário cooperativo genérico 65

Figura 28- Grade de um circuito com duas entradas (cinza) e uma saída (preto). Os números indicam as posições (o ponto à esquerda e abaixo do número) onde novas células de QCA podem ser posicionadas. 66

Figura 29- Algoritmo de construção do circuito. 68

Figura 30- Criação passo a passo da topologia do circuito de acordo com os cromossomos utilizados.

Figura 31- Exemplo do problema de ótimo local quando a função de avaliação só leva em consideração o número de acertos. 71

Figura 32- Média dos melhores indivíduos por geração..................................... 74

Figura 33- Melhor circuito OU de quatro entradas evoluído pelo AG................. 74

Figura 34 - Circuito OU proposto na literatura................................................... 75

Figura 35- Média dos melhores indivíduos por geração.................................... 77

Figura 36- Topologia do melhor multiplexador encontrado pelo AG, contendo 14 células. Os tons de cinza representam as zonas de clock. 78

Figura 37 - Topologia do multiplexador sugerido por Lent, contendo 21 células.

Os tons de cinza representam as zonas de clock 79

Figura 38- Média dos melhores indivíduos por geração. 80

Figura 39- Topologia do melhor Ou-exclusivo encontrado pelo AG. Os tons de 
cinza representam as zonas de clock. 81

Figura 40- Topologia do Ou-exclusivo proposto na literatura........................... 81

Figura 41- Topologia do somador completo. Partes vermelhas e verdes serão substituídas por partes menores. .83

Figura 42- Topologia do novo circuito do somador completo........................... 84

Figura 43- Média dos melhores indivíduos por geração...................................... 86

Figura 44- Topologia do novo somador completo. .......................................... 86

Figura 45- Células rotacionadas lado a lado. ......................................................97

Figura 46- Uma célula convenciona e uma célula rotacionada lado a lado.......... 98

Figura 47- Células convencionais distanciadas de meia célula........................... 98

Figura 48- Células rotacionadas distanciadas de meia célula. ............................98

Figura 49- Uma célula convenciona e uma célula rotacionada distanciadas de

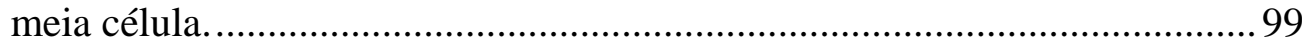

Figura 50- Células convencionais distanciadas de uma célula............................ 99

Figura 51- Células rotacionadas distanciadas de uma célula...........................100

Figura 52- Uma célula convenciona e uma célula rotacionada distanciadas de

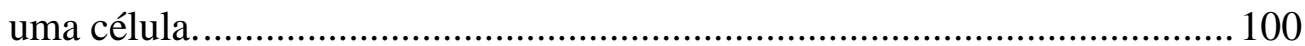

Figura 53- Células convencionais em diagonal............................................. 101

Figura 54- Células rotacionadas em diagonal. ................................................ 101

Figura 55- Uma célula convenciona e uma célula rotacionada em diagonal. ... 102

Figura 56-Células convencionais em diagonal............................................. 102

Figura 57- Células rotacionadas em diagonal distanciadas............................... 103

Figura 58- Uma célula convenciona e uma célula rotacionada em diagonal distanciadas. 103

Figura 59- As posições das células com a “kink energy” positiva. Os quadrados pontilhados indicam as posições onde a “kink energy” é negativa devido a

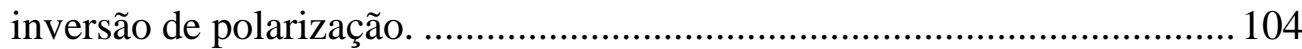

Figura 60- Células convencionais em meia diagonal........................................105

Figura 61- Células rotacionadas em meia diagonal......................................... 105

Figura 62- Uma célula convenciona e uma célula rotacionada em meia diagonal distanciadas 106

Figura 63- As posições das células com a “kink energy” positiva. Os quadrados pontilhados indicam as posições onde a "kink energy" é negativa devido a 
inversão de polarização. 106

Figura 64- Células convencionais em meia diagonal distanciadas. .................. 106

Figura 65- Células rotacionadas em meia diagonal. 107

Figura 66- Uma célula convenciona e uma célula rotacionada em meia diagonal distanciadas. 107

Figura 67- As posições das células com a "kink energy" negativa. Os quadrados pontilhados indicam as posições onde a “kink energy” é positiva devido a inversão de polarização. 108

Figura 68- Representação do inversor. 109

Figura 69- As simulações do inversor para os dois valores de entrada possíveis. 109

Figura 70- Representação de um circuito com dois fios cruzando em um plano. 110

Figura 71- Simulação de dois fios cruzando em um plano. Todos os conjuntos de entradas

Figura 72- Representação de um majority gate. 111

Figura 73- Simulação de um majority gate. Todos os conjuntos de entradas... 112 Figura 74- Simulação de um majority gate. Todos os conjuntos de entradas... 113 Figura 75- Representação de um majority gate com as entradas partindo da esquerda.

Figura 76- Simulação de um outro majority gate. Todos os conjuntos de entradas.

Figura 77- Representação de um majority gate, com zonas de clock. Os sinais fluem da esquerda para a direita. 115

Figura 78- Simulação passo a passo do majority gate com zonas de clock........ 117

Figura 79- Representação de um somador completo com três entradas. 117

Figura 80- Simulação passo a passo de um somador completo. 119

Figura 81- Representação de um somador completo serial com duas entradas. 120 Figura 82- Simulação passo a passo de um somador completo serial................122 Figura 83- Melhor circuito OU de quarto entradas evoluído pelo AG.............. 123

Figura 84- Simulações da porta OU sintetizada.............................................. 124

Figura 85- Melhor Multiplexador sintetizado. .................................................. 124

Figura 86- Simulações do Multiplexador sintetizado......................................... 125 
Figura 87- Topologia do melhor Ou-exclusivo encontrado pelo AG. Os tons de cinza representam as zonas de clock........................................................ 126

Figura 88- Simulações da porta OU-Exclusivo sintetizada............................. 126

Figura 89- Topologia do novo circuito do somador completo......................... 127

Figura 90- Simulações do Somador completo 1 sintetizado. ............................. 128

Figura 91- Topologia do novo circuito do somador completo - alternativa 2. . 128

Figura 92- Simulações do Somador completo 1 sintetizado. 130 


\section{Lista de tabelas}

Tabela 1- Trabalhos em nanotecnologia utilizando Inteligência Computacional. 32

Tabela 2-Simuladores de QCA já desenvolvidos............................................... 48

Tabela 3- "Kink energy" para todas as possíveis posições que respeitem o raio de

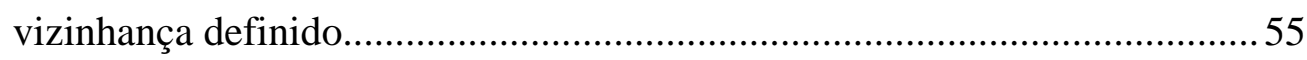

Tabela 4- O estado de polarização de cada célula, a cada passo da rede neural. 60

Tabela 5- Comparação dos dados de 10 experimentos....................................... 73

Tabela 6- Comparação dos dados de 10 experimentos....................................... 77

Tabela 7- Comparação entre a busca aleatória e o AG. 85 